\title{
Effect of fuzzy-membership function types on the search capability of interactive search algorithm
}

\author{
A. Mortazavi*iD \\ Ege University, Graduate School of Natural and Applied Sciences, Izmir, Turkey
}

\begin{abstract}
Fuzzy logic is widely used as the fundamental platform for the many artificial intelligence-based systems like control of subways, air conditioners, transmission systems, facial pattern recognition, antiskid braking systems. In the theory of fuzzy set, membership functions (MFs) provide an infrastructure for determining the degree of truth in a fuzzy model. So, they can play important role in the performance of these logical mechanisms. Different types of MF can be defined to fuzzification-defuzzification process (i.e. converting Boolean input to a fuzzy output and vise-versa). They are categorized and named based on the shape of their diagrams as triangular, Gaussian, trapezoidal and so forth. In the current study the effect of MF's type on the search capability of Interactive Search Algorithm (ISA) is assessed. To meet this aim, a metaheuristic technique that are known as Interactive Search Algorithm and strengthen with fuzzy adjustment mechanism is tested on solving a number of benchmark problems involving different types of MFs. The results indicate that due to stochastic essence of metaheuristic approaches the type of MF does not seriously affect the search capability of this technique. Therefore, it can be stated that the MFs should be selected based on the simplicity criterion. In other words, the simplest MF which provides the designers requirements can be the best choice.
\end{abstract}

\section{Keywords}

Metaheuristic methods; Fuzzy logic; Membership functions

Received: 19 October 2019; Accepted: 25 November 2019

ISSN: 2630-5763 (online) C 2019 Golden Light Publishing All rights reserved.

\section{Introduction}

Most of the engineering optimization problems have a complex search space and generally finding a continuous and differentiable objective function for this class of problems is very difficult or mostly unattainable [1]. So, applying the gradient-based optimization methods which require the continuous (or at least partially continuous) objective function(s) and its different order gradients mostly is very challenging or even impossible in some cases [2-5]. Consequently, alternative optimization tool is required for such a complex problem.

* Corresponding author

Email: ali.mortazavi.phd@gmail.com
Metaheuristic algorithms are the non-gradient based algorithms which do not require any continuous objective function and/or its gradients. These methods are generally put forward a simple stochastic mathematic algorithm which is inspired from the natural phenomenon like physical and natural rules, social behaviors or collaboration in the fish and/or birds colonies [6-23]. In addition of these specifications their ease to implementation makes them applicable in solving a wide range of optimization problems [24-28].

One can list some of these methods as, differential-based harmony search algorithm for the 
optimization of continuous problems, Search Group Algorithm (SGA) [23], Virus optimization algorithm (VOA) [14], Multi-strategy adaptive particle swarm optimization [18]. Each of these methods based on the governing conditions of the selected optimization problems has its own strengths and weaknesses. One of the most important factors which affects the search capability of the metaheuristic technique is its capability to adequately and properly perform exploitation and exploration search behaviors. In this regard, an algorithm that can adapt itself more closely to the dominant conditions of the problem shows higher search capability.

The metaheuristics mostly uses internal adjustable parameters to perform this action. The appropriate values for these parameters can be set via test and training sets or by applying auxiliary tuner modulus. In the current investigation the fuzzy mechanism is used to adjust the internal parameters of the Interactive Search Algorithm (ISA) introduced by Mortazavi et al. [29]. This algorithm uses two main internal adjustable parameters to balance the search behavior of the algorithm. Similar to several other optimization algorithms these internal terms highly affect the search capability of the technique. To maximize the search performance of the algorithm these parameters should be adjusted for desired class of problem(s) through the proper sensitivity analyses [30]. However setting up the internal parameters for the specific type(s) of problem may cause the technique to decrease its performance on solving other type of problems [31]. To prevent this shortcoming, the smart decision-making systems can be integrated to these methods. In this regard the fuzzy logic is applied as an auxiliary system which permanently monitors the optimization process and adaptively sets the proper values for internal coefficients of the proposed ISA method. To form such system different membership functions (MFs) can be implemented. The current study investigates the effect of different type of membership functions on the search performance of the ISA method. In this regard, six well known membership functions named as Triangular, Trapezoid, two variants of Gaussian, Gbellmf, and Sigmoidal are selected to test the effect of the membership functions on the search performance of the ISA algorithm. There several mathematical functions and mechanical problems are chosen as benchmark problems. At the following firstly the applied ISA method is briefly described and then the membership functions are explained. In the next section the numeric tests are perfumed and results are reported. Finally, in the conclusion part the attained outcomes are instructed.

\section{Interactive search algorithm (ISA)}

Interactive Search Algorithm (ISA) is the hybrid optimization algorithm introduced by Mortazavi et al. [29]. This method utilizes two different patterns to navigate agent in the search space. They are named as tracking and interacting patterns. In the tracking pattern $i$ th each agent $\left(\boldsymbol{X}_{i}\right)$ is updated considering three specific agent as weighted agent $\left(\boldsymbol{X}^{W}\right)$, the global best agent $\left(\boldsymbol{X}^{G}\right)$ and the prior best position stored in the memory of randomly selected another agent $\left(\boldsymbol{X}_{j}^{P}\right)$. In the interacting pattern, the current particle updates its position via pairwise data sharing with other agents in the colony. In each iteration agents based on their tendency factor select either interacting or tracking paradigm. The tendency factor $\left(\tau_{i}\right)$ in the standard ISA is designated to each agent randomly from $[0,1]$ interval with normal distribution. It should be noted that the tracking and interacting patterns provide the global and local search requirements of the ISA algorithm, respectively. based on given definitions the proposed ISA algorithm mathematically is defined as below:

$$
\left.\begin{array}{rl}
\text { if } \tau_{i} \geq & 0.3 \\
{ }^{t+1} \mathbf{v}_{i}= & w_{i} \times{ }^{t} \mathbf{v}_{i}+\operatorname{diag}\left(\boldsymbol{\varphi}_{1 i} \otimes\left({ }^{t} \mathbf{X}_{j}^{P}-{ }^{t} \mathbf{X}_{i}\right)\right) \\
& +\operatorname{diag}\left(\boldsymbol{\varphi}_{2 i} \otimes\left({ }^{t} \mathbf{X}^{G}-{ }^{t} \mathbf{X}_{j}^{P}\right)\right) \\
& +\operatorname{diag}\left(\boldsymbol{\varphi}_{3 i} \otimes\left({ }^{t} \mathbf{X}^{w}-{ }^{t} \mathbf{X}_{j}^{P}\right)\right)
\end{array}\right\} \text { (Tracking) }
$$


$\left.\begin{array}{l}\text { if } \tau_{i}<0.3 \\ { }^{t+1} \mathbf{v}_{i}=\operatorname{diag}\left(\mathbf{r}_{i}^{T} \otimes\left(\mathbf{X}_{i}-\mathbf{X}_{j}\right)\right) \text { if } f\left(\mathbf{X}_{i}\right)<f\left(\mathbf{X}_{j}\right) \\ { }^{t+1} \mathbf{v}_{i}=\operatorname{diag}\left(\mathbf{r}_{i}^{T} \otimes\left(\mathbf{X}_{j}-\mathbf{X}_{i}\right)\right) \text { if } f\left(\mathbf{X}_{i}\right)>f\left(\mathbf{X}_{j}\right)\end{array}\right\}$ (Interacting)

$$
{ }^{t+1} \mathbf{X}_{i}={ }^{t} \mathbf{X}_{i}+{ }^{t+1} \mathbf{v}_{i}
$$

where $\boldsymbol{X}_{i}$ is the $i$ th agent (i.e. vector) in the colony, $\boldsymbol{X}_{i}=\left[x_{i}^{1}, x_{i}^{2}, x_{i}^{3}, \ldots, x_{i}^{n d}\right]$ and " $t$ " and " $t+l$ " superscripts are the current and updated conditions, respectively. Also, $\otimes$ indicates the Kronecker multiplier and $\operatorname{diag}($ ) returns a vector holds any square matrix's diagonal elements. To provide more clarity on the mechanism of this multiplication assume two $n$-dimensional vectors namely $\mathbf{a}=\left[a_{1}, a_{2}, \ldots, a_{n}\right]$ and $\mathbf{b}=\left[b_{1}, b_{2}, \ldots, b_{n}\right]$, for these vectors $\operatorname{diag}(\mathbf{a} \otimes \mathbf{b})$ returns an $n$ dimensional vector which their elements are $a_{l} b_{l}$ where $1 \leq l \leq n$. Also, $1 \leq i, j \leq n p, i \neq j$ and $n p$ indicates the size of population. For $i$ th agent $\varphi_{k i}=C_{k} \times \operatorname{rand}_{k i}$ for $k \in\{1,2,3\}$ and $C_{1}=-\left(\varphi_{2 i}+\right.$ $\left.\varphi_{3 i}\right), C_{2}=2, C_{3}=1$ are coefficients of acceleration, and $\operatorname{rand}_{k i}$ are vectors hold random numbers selected from the $[0,1]$ interval. ${ }^{t} \mathbf{X}^{w}$ is the weighted agent which can be calculated from (1), and $\boldsymbol{X}^{P}$ is a matrix keeps the previous best found positions of all agents. ${ }^{t} \mathbf{X}_{j}^{P}$ is the arbitrarily selected agent from the matrix of $\boldsymbol{X}^{P}$ with uniform probability. The weighted agent $\left(\boldsymbol{X}^{W}\right)$ is the weighted average of all available agents which is defined as follow:

$$
\begin{aligned}
& \mathbf{X}^{W}=\sum_{i=1}^{M} \bar{c}_{i}^{w} \mathbf{X}_{i}^{P} \\
& \bar{c}_{i}^{w}=\left(\hat{c}_{i}^{w} / \sum_{i=1}^{M} \hat{c}_{i}^{w}\right) \\
& \hat{c}_{i}^{w}=\frac{\max _{1 \leq k \leq M}\left(f\left(\mathbf{X}_{k}^{P}\right)\right)-f\left(\mathbf{X}_{i}^{P}\right)+\varepsilon}{\max _{1 \leq k \leq M}\left(f\left(\mathbf{X}_{k}^{P}\right)\right)-\min _{1 \leq k \leq M}\left(f\left(\mathbf{X}_{k}^{P}\right)\right)+\varepsilon} \quad i=1,2, \ldots, M
\end{aligned}
$$

where $\mathbf{M}$ is the number of all particles, $\mathbf{x}^{W}$ denotes the position vector of the weighted particle, $\hat{c}_{i}^{w}$ is the coefficient shows influence of each particle on the weighted particle, $\mathrm{f}($.$) is the objective function$ of the optimization problem, $\underset{1 \leq k \leq M}{\max }\left(f\left(\mathbf{X}_{k}^{P}\right)\right)$ and $\min _{1 \leq k \leq M}\left(f\left(\mathbf{X}_{k}^{P}\right)\right)$, respectively, are the worst and best objective values among all particles, $\varepsilon$ is a small positive number avoids division by zero.

ISA uses the improved fly-back (IFB) technique to handle any problem's constraints [29]. On contrary of the conventional penalty approach(s), the improved fly-back approach is an ad-hoc parameters free approach. This method is described in details in reference [1] however to give more illustrations this method is described concisely. The proposed IFB consists of three main steps [29]:

(i) The problem's constraints are divided into two categories as characteristic (those need to objective function evaluation to check their violations) and numeric (those do not need to objective function evaluation to check their violations).

(ii) Each component of the current agent which violates any numeric constraints is replaced with corresponding components of the weighted agent.

(iii) If updated agent is feasible (does not violate the characteristic constraints), and provides better objective value it is hold and matrix of $\boldsymbol{X}^{P}$ is updated, but if not, it is rest to its prior best position stored in $\boldsymbol{X}^{P}$.

\section{Fuzzy adjusting the tendency factor}

As described in the previous section the tendency factor $\left(\tau_{i}\right)$ determines the search pattern for each agent. In the standard version of ISA this factor is randomly generated, but to determine it more properly, it can be designated via a fuzzy 
mechanism. Since the focused of the current work is not to addressed the definition of the fuzzy mechanism to prevent any prolongation the details about this fuzzy system is not provided. The interested readers can find this mechanism definition and work process in reference [32]. The membership function plays determinative role in the performance of the fuzzy system, thus the different types of these functions are assessed. In this regard, initially a definition of these membership functions are explained in following.

\subsection{1 Membership function}

The membership function of a fuzzy set is a generalization form of the indicator function in the conventional sets. In the fuzzy logic, it represents the degree of truth as an extension of valuation. It should be noted that probabilities and degrees of truth are two different definitions and they are conceptually distinct. The fuzzy truth signifies membership in unclearly defined sets, but not likelihood of some conditions or events. Zadeh [32] introduced the membership functions for the first time in the literature. A fuzzy set $\tilde{A}$ in the universe of information $U$ can be defined as a set of ordered pairs and it can be represented mathematically as below:

$$
\tilde{A}=\left\{\left(y, \mu_{\tilde{A}}(y)\right) \quad y \in U\right\}
$$

where $\mu_{\tilde{A}}(\cdot)=$ membership function of $A$ while $\mu_{\tilde{A}}$ $(\cdot) \in[0,1]$. The membership function $\mu_{\tilde{A}}(\cdot)$ maps $U$ to the membership space $M$. Different types of membership functions involved in the current work are addressed in Fig. 1.

\section{Numeric tests}

This section is devoted to testing the effect of membership functions on the search behavior of the proposed ISA method. In this regard, two group of examples are selected. First, non-constrained mathematical functions and second, the constrained mechanical problems. The results are reported through illustrative tables.

\subsection{Mathematical benchmark functions}

In this section five well-known benchmark functions taken into consideration. These functions are shifted, while the fourth one is also rotated. These functions can change proposed optimization algorithm from different aspects. For instance, the first function has a smoother search space while the third one has a very noisy search domain. These functions are chosen in such a way that all membership functions be tested on both simple and complex search domains. The corresponding functions are schematically plotted and their formulations tabulated in Table 1. The dimension of these functions is taken as $\mathrm{D}=60$ in the current work. Maximum number of iterations is set as $10000 * \mathrm{D}$. To prevent any premature convergence, each problem runs for 30 times.

The results obtained from the numerical experiments are addressed in the Table 2. The successful rate (SR) and standard deviation (Std.) for all test functions are reported in this table. SR is defined as percentage of runs which algorithm can achieve the predefined accuracy level within the maximum allowable number of iterations. The accuracy level is set as 1E-2 for all functions in this investigation. Based on the results presented in Table 2, it can be worthy expressed that the types of membership function do not seriously affect the search performance of the algorithm. Therefore, the triangular MF seems to have top priority for implementation in such problems, since it is the simplest membership function which can provide the requirements.

\subsection{Welded beam constrained problem}

The welded beam problem shown in Fig. 2 is examined as the constrained optimization problem. The objective of this problem is the total cost of the welded beam which should be minimized involving seven different constraints based on the deflection $(\delta)$, shear stress $(\tau)$, normal stress $(\sigma)$, etc. The welding's properties $(l, h)$ and cross-section $(b, t)$ specifications are taken into account as the design variables of the problem. 


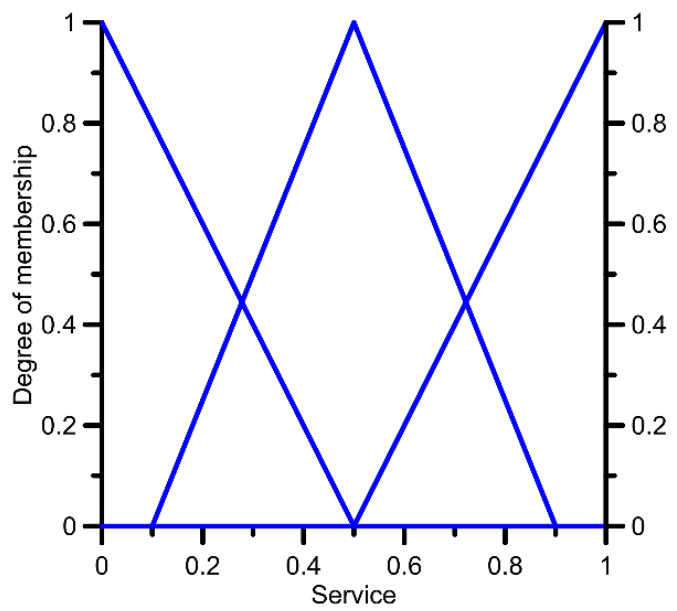

a. MF1: Triangular

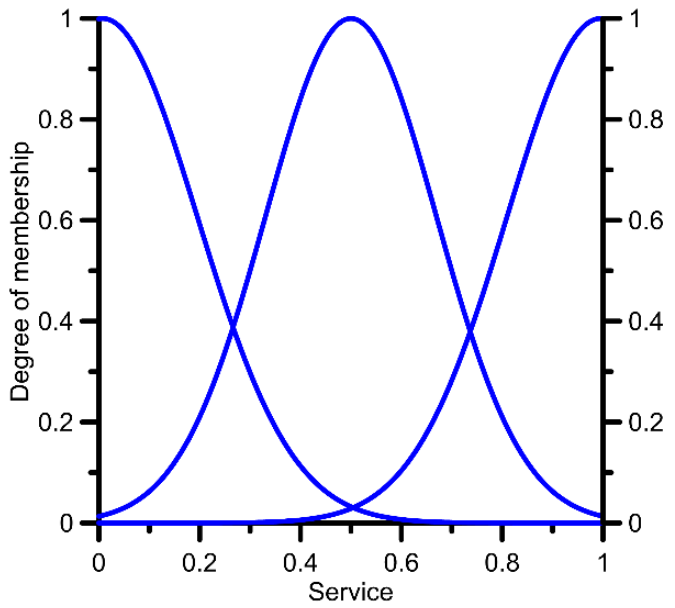

c. MF3: Gaussian

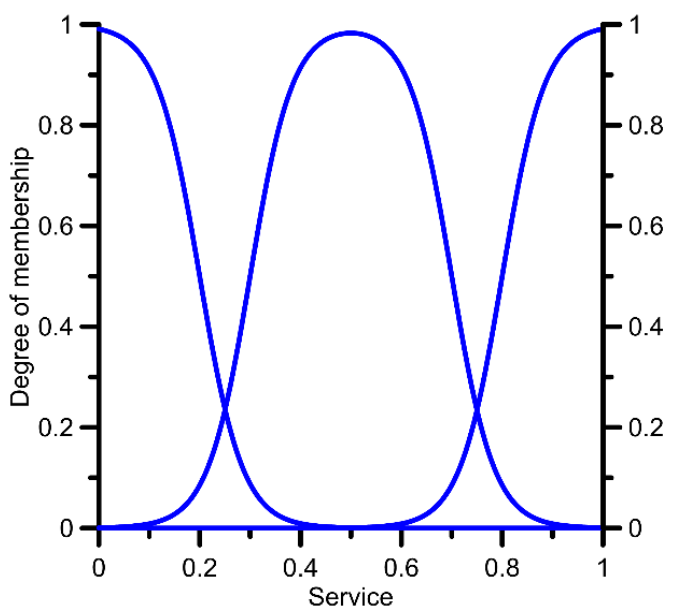

e. MF5: Sigmoidal

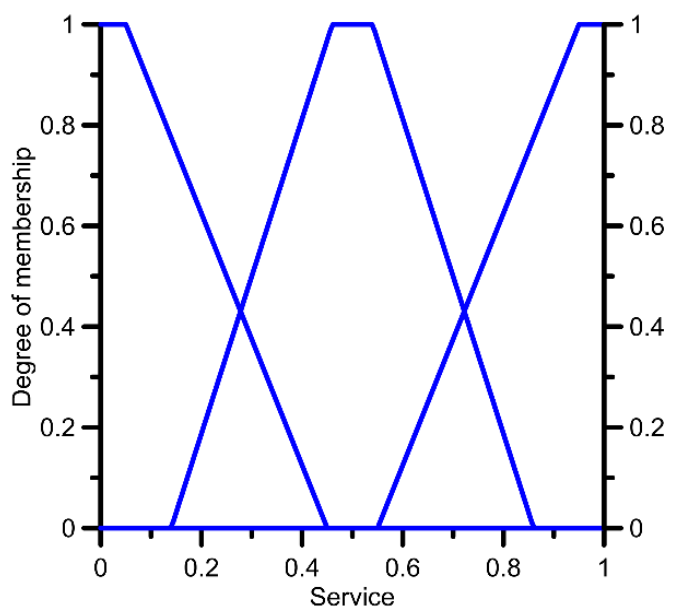

b. MF2: Trapezoid

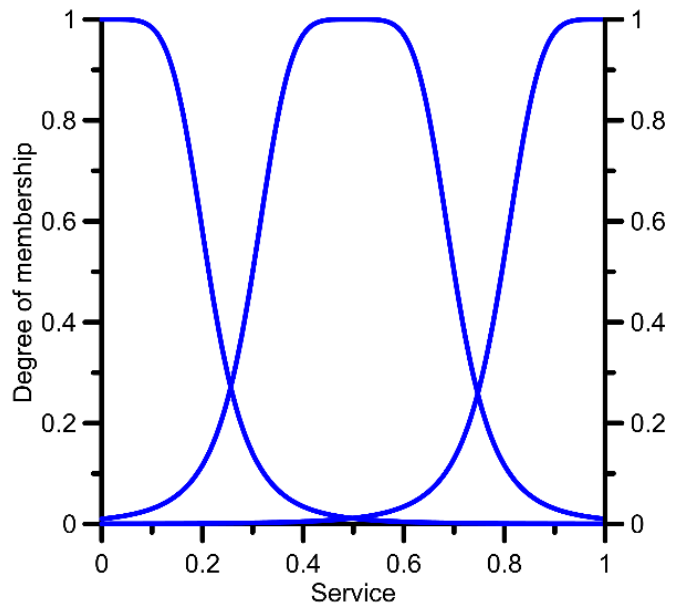

d. MF4: Gbellmf

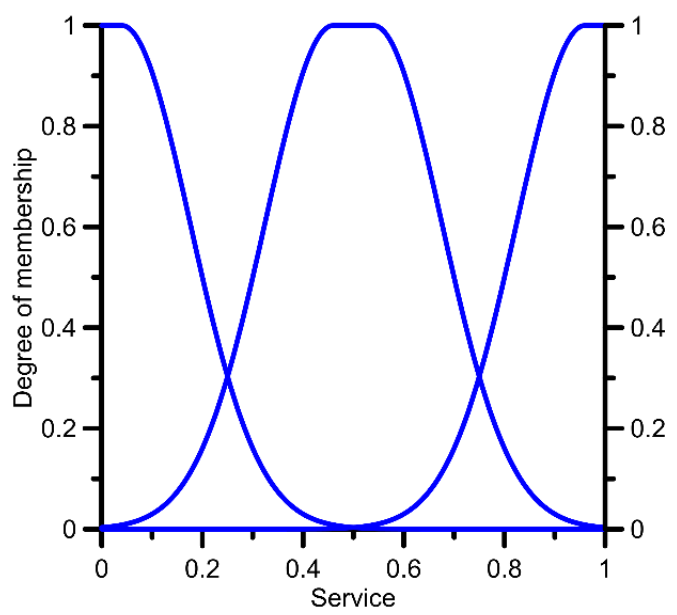

f. MF6: Gaussian2

Fig. 1. Different types membership functions (MFs) 
Table 1. The benchmark functions properties

\begin{tabular}{ll}
\hline Function & Plot \\
\hline F1: Shifted & \\
Sphere f1 &
\end{tabular}

F2: Shifted Schwefel's 1.2

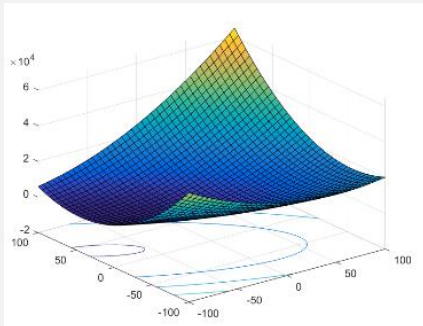

F3: Shifted Schwefel's 1.2 with Noise in Fitness

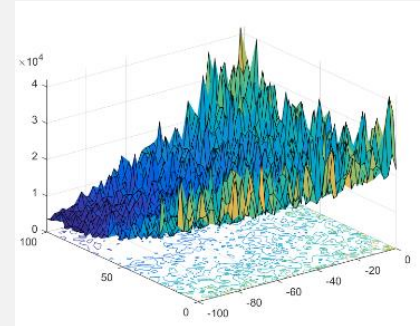

F4: Shifted rotated Weierstrass

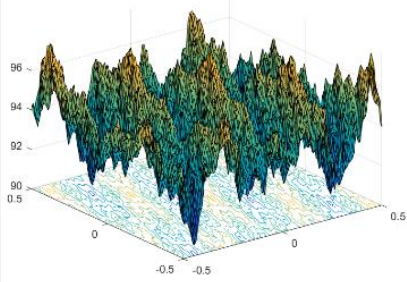

F5: Shifted Rastrigin's

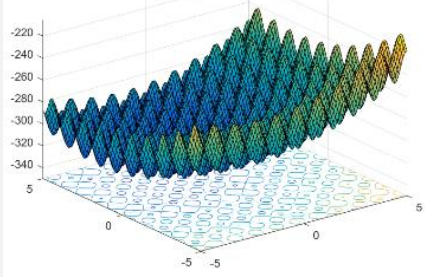

$F_{1}(\mathbf{x})=\sum_{i=1}^{D} z_{i}^{2}+f \_$bias,

$\mathbf{Z}=\mathbf{X}-\mathbf{O}, \mathbf{X}=\left[x_{1}, x_{2}, \ldots, x_{D}\right]$

$\mathbf{X} \in[-100,100]$

$F_{2}(\mathbf{x})=\sum_{i=1}^{D}\left(\sum_{j=1}^{i} z_{j}^{2}\right)+f_{-}$bias,

$\mathbf{Z}=\mathbf{x}-\mathbf{0}, \mathbf{x}=\left[x_{1}, x_{2}, \ldots, x_{D}\right]$

$\mathbf{x} \in[-100,100]$

$f(\mathbf{X})=\left(\sum_{i=1}^{D}\left(\sum_{j=1}^{i} z_{j}\right)^{2}\right)(1+0.4|N(0,1)|)-450$

$\mathbf{Z}=\mathbf{X}-\mathbf{0}, \quad \mathbf{X}=\left[x_{1}, x_{2}, \ldots, x_{D}\right], \quad \mathbf{X} \in[-100,100]$

$F_{4}(\mathbf{x})=\sum_{i=1}^{D}\left(\sum_{k=1}^{k_{\max }}\left[a^{k} \cos \left(2 \pi b^{k}\left(z_{i}+0.5\right)\right)\right]\right)$

$-D \sum_{k=1}^{k_{\max }}\left[a^{k} \cos \left(2 \pi b^{k}\left(z_{i}+0.5\right)\right)\right]+f_{-}$bias,

$a=0.5, b=3, k_{\max }=20$,

$\mathbf{Z}=(\mathbf{x}-\mathbf{0}) \times \mathbf{M}, \mathbf{x}=\left[x_{1}, x_{2}, \ldots, x_{D}\right]$

$\mathrm{x} \in[-0.5,+0.5]$

$F_{5}(\mathbf{x})=\sum_{i=1}^{D}\left(\mathbf{z}_{i}^{2}-10 \cos \left(2 \pi \mathbf{z}_{i}\right)+10\right)+f \_$bias,

$\mathbf{Z}=(\mathbf{x}-\mathbf{0}) \times \mathbf{M}, \mathbf{x}=\left[x_{1}, x_{2}, \ldots, x_{D}\right]$

$\mathbf{x} \in[-5,+5]$ 
Table 2. The results for different membership functions

\begin{tabular}{llllllll}
\hline $\begin{array}{l}\text { Test } \\
\text { Functions }\end{array}$ & & MF1 & MF2 & MF3 & MF4 & MF5 & MF6 \\
\hline F1 & Mean & -12.98 & -12.93 & -12.91 & -12.97 & -12.94 & -12.98 \\
& Std. & $8.12 \mathrm{E}-8$ & $8.01 \mathrm{E}-8$ & $8.19 \mathrm{E}-8$ & $8.08 \mathrm{E}-8$ & $8.58 \mathrm{E}-8$ & $8.41 \mathrm{E}-8$ \\
& SR & 100 & 100 & 100 & 100 & 100 & 100 \\
F2 & Mean & 12.81 & 12.88 & 12.82 & 12.80 & 12.82 & 12.86 \\
& Std. & $6.22 \mathrm{E}-8$ & $6.87 \mathrm{E}-8$ & $7.01 \mathrm{E}-8$ & $6.55 \mathrm{E}-8$ & $6.41 \mathrm{E}-8$ & $6.66 \mathrm{E}-8$ \\
& SR & 100 & 100 & 100 & 100 & 100 & 100 \\
F3 & Mean & -2.28 & -2.35 & -2.33 & -2.33 & -2.30 & -2.29 \\
& Std. & $5.02 \mathrm{E}-3$ & $5.51 \mathrm{E}-3$ & $6.82 \mathrm{E}-3$ & $5.77 \mathrm{E}-3$ & $5.14 \mathrm{E}-3$ & $5.59 \mathrm{E}-3$ \\
& SR & 73 & 70 & 73 & 80 & 73 & 80 \\
F4 & Mean & 2.21 & 2.23 & 2.20 & 2.22 & 2.22 & 2.24 \\
& Std. & $7.14 \mathrm{E}-1$ & $7.11 \mathrm{E}-1$ & $7.09 \mathrm{E}-1$ & $7.88 \mathrm{E}-1$ & $7.07 \mathrm{E}-1$ & $7.62 \mathrm{E}-1$ \\
& SR & 0 & 0 & 0 & 0 & 0 & 0 \\
& Mean & 2.01 & 2.01 & 2.05 & 2.12 & 2.09 & 2.08 \\
& Std. & $8.10 \mathrm{E}-1$ & $8.08 \mathrm{E}-1$ & $8.12 \mathrm{E}-1$ & $8.11 \mathrm{E}-1$ & $8.57 \mathrm{E}-1$ & $8.02 \mathrm{E}-1$ \\
& SR & 0 & 0 & 0 & 0 & 0 & 0 \\
\hline
\end{tabular}

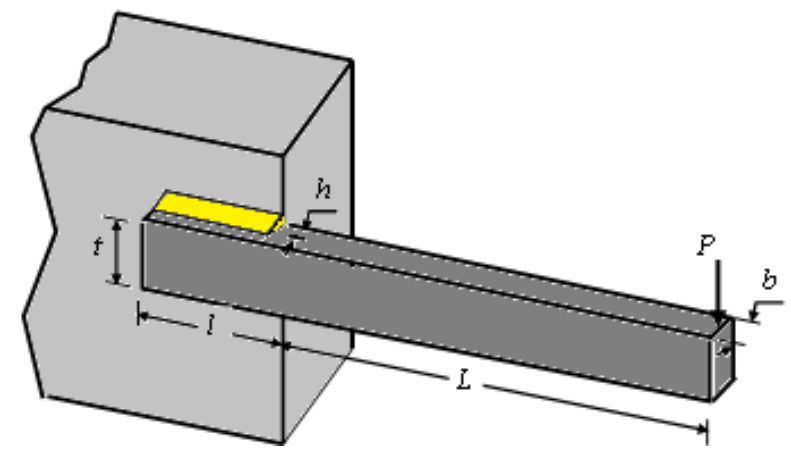

Fig. 2. The welded beam problem

$$
\mathbf{X}=\left\{x_{1}, x_{2}, x_{3}, x_{4}\right\}
$$

$\operatorname{cost}(\mathbf{X})=1.10471 x_{1}^{2} x_{2}+0.04811 x_{3} x_{4}\left(14+x_{2}\right)$

Subject to

$$
\begin{aligned}
& g_{1}(x)=\tau(x)-\tau_{\max } \leq 0 \\
& g_{2}(x)=\sigma(x)-\sigma_{\max } \leq 0 \\
& g_{3}(x)=x_{1}-x_{4} \leq 0
\end{aligned}
$$

$$
\begin{aligned}
& g_{4}(x)=0.10471 x_{1}^{2}+0.04811 x_{3} x_{4}\left(14+x_{2}\right)-5 \leq 0 \\
& g_{5}(x)=0.125-x_{1} \leq 0 \\
& g_{6}(x)=\delta(x)-\delta_{\max } \leq 0 \\
& g_{7}(x)=p-p_{c}(x) \leq 0 \\
& 0.1 \leq x_{1} \leq 2, \quad 0.1 \leq x_{2} \leq 10, \quad 0.1 \leq x_{3} \leq 10, \quad 0.1 \leq x_{4} \leq 2
\end{aligned}
$$


where

$$
\begin{aligned}
& \tau(x)=\sqrt{\left(\tau^{\prime}\right)^{2}+2 \tau^{\prime} \tau^{\prime \prime} \frac{x_{2}}{2 R}+\left(\tau^{\prime \prime}\right)^{2}} \\
& \tau^{\prime}=\frac{P}{\sqrt{2} x_{1} x_{2}} \quad \tau^{\prime \prime}=\frac{M R}{J} \quad M=P\left(\mathrm{~L}+\frac{x_{2}}{2}\right) \\
& R=\sqrt{\frac{x_{2}^{2}}{4}+\left(\frac{x_{1}+x_{3}}{2}\right)^{2}} \\
& J=2\left\{\sqrt{2} x_{1} x_{2}\left[\frac{x_{2}^{2}}{12}+\left(\frac{x_{1}+x_{3}}{2}\right)^{2}\right]\right\} \\
& \sigma(x)=\frac{6 P L}{x_{4} x_{3}^{2}} \quad \delta(x)=\frac{4 P L^{3}}{E x_{3}^{3} x_{4}} \\
& P_{c}(x)=\frac{4.013 \sqrt{E\left(x_{3}^{2} x_{4}^{6} / 36\right)}}{L^{2}}\left(1-\frac{x_{3}}{2 L} \sqrt{\frac{E}{4 G}}\right)
\end{aligned}
$$

in which $P=6000 \mathrm{lb}, L=14$ in., $E=30 \times 10^{6} \mathrm{psi}$, $G=12 \times 10^{6} \mathrm{psi}, \tau_{\max }=13,600 \mathrm{psi}, \sigma_{\max }=30,000 \mathrm{psi}$, and $\delta_{\max }=0.25 \mathrm{in}$.

The results obtained from the numerical investigations performed for this example with different membership functions is tabulated in Table 3. As can be seen from the results given in Table 3, variation of the membership function type has not a significant affect on the search capability of the algorithm.

\subsection{A gear system}

The gear system shown in Fig. 3 is evaluated as the last example in the current study. The objective of current example is to minimize the gear size ratio in the given gear mechanism of a train system. The objective function of this problem is mathematically illustrated as follows:

Minimize $f(\mathbf{X})=\left(\frac{1}{6.931}-\frac{x_{1} x_{2}}{x_{3} x_{4}}\right)^{2}$

subject to:

$12 \leq x_{i} \leq 60 \quad i=1,2,3,4$

where parameters of $x_{1}, x_{2}, x_{3}$ and $x_{4}$ give the number of gear teeth and range of them also $x_{i} \in[12,60], i=1,2,3,4$. The proposed ratio is formulated as follows:

$$
\text { The gear ratio }=\frac{\text { angular velocity of output shaft }}{\text { angular velocity of input shaf }}
$$

The results obtained with utilizing different membership functions are presented in Table 4. Studying the results given in Table 4 , it can be argued that similar to prior examples, the membership function type has no any particular effect in the search capability of the algorithm.

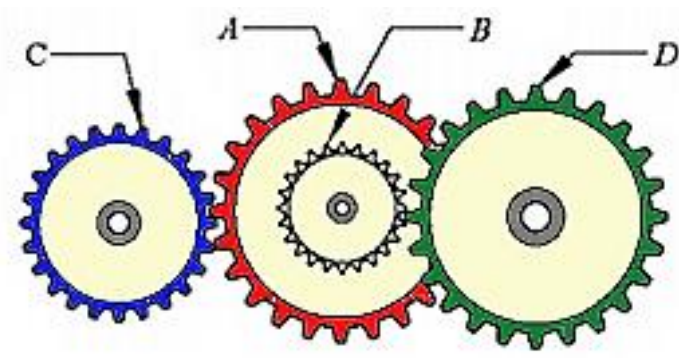

Fig 3. The gear system

Table 3. Welded beam example's results for different membership functions

\begin{tabular}{lllllll}
\hline Value & MF1 & MF2 & MF3 & MF4 & MF5 & MF6 \\
\hline Mean & 1.724856 & 1.724858 & 1.724856 & 1.724857 & 1.724856 & 1.724857 \\
Std. & 0.00 & $1.20 \mathrm{E}-7$ & 0.00 & $1.04 \mathrm{E}-7$ & 0.00 & $1.98 \mathrm{E}-8$ \\
\hline
\end{tabular}

Table 4. Gear system results

\begin{tabular}{lllllll}
\hline Value & MF1 & MF2 & MF3 & MF4 & MF5 & MF6 \\
\hline Best & $2.70 \mathrm{E}-12$ & $2.70 \mathrm{E}-12$ & $2.70 \mathrm{E}-12$ & $2.70 \mathrm{E}-12$ & $2.70 \mathrm{E}-12$ & $2.70 \mathrm{E}-12$ \\
Std. & $4.5 \mathrm{E}-12$ & $4.51 \mathrm{E}-12$ & $4.58 \mathrm{E}-12$ & $4.51 \mathrm{E}-12$ & $4.55 \mathrm{E}-12$ & $4.56 \mathrm{E}-12$ \\
\hline
\end{tabular}




\section{Conclusion}

The current study deals with evaluating the effect of membership function types on the performance of the fuzzy-reinforced metaheuristic algorithms. In this regard, the Interactive Search Algorithm (ISA) is selected as the pilot optimization approach. The six prevalent types of membership functions named as triangular, trapezoidal, Gaussian, Sigmoidal and Bell are considered to adjust the fuzzy mechanism. It should be noted, among the all studied membership functions triangular membership function is the simplest one due to its linear approach. Five different none-constrained mathematical functions and two constrained mechanical problems are assessed as the benchmark problems. Achieved outcomes are summarized in the illustrative tables. The attained results show that types of membership function do not seriously affect the performance of the fuzzy module of the algorithm. Consequently, to define a fuzzy mechanism, picking the simplest membership function which can meet the requirements would be preferred.

\section{References}

[1] A. Mortazavi, V. Toğan, Simultaneous size, shape, and topology optimization of truss structures using integrated particle swarm optimizer, Structural and Multidisciplinary Optimization, 54 (2016) 715736.

[2] A. Mortazavi, V. Toğan, A. Nuhoğlu, An integrated particle swarm optimizer for optimization of truss structures with discrete variables, Structural Engineering and Mechanics, 61 (2017) 359-370.

[3] Y. Zhang, D. Gong, J. Cheng, Multi-Objective Particle Swarm Optimization Approach for CostBased Feature Selection in Classification, IEEE/ACM Transactions on Computational Biology and Bioinformatics, 14 (2017) 64-75.

[4] Y. Zhang, D.-w. Gong, J.-h. Zhang, Robot path planning in uncertain environment using multiobjective particle swarm optimization, Neurocomputing, 103 (2013) 172-185.

[5] A.A. El-Sawy, Z.M. Hendawy, M.A. El-Shorbagy, Reference Point Based TR-PSO for MultiObjective Environmental/Economic Dispatch, Applied Mathematics, Vol.04No.05 (2013) 11.
[6] A. Mortazavi, V. Toğan, A. Nuhoğlu, Weight minimization of truss structures with sizing and layout variables using integrated particle swarm optimize, Journal of Civil Engineering and Management, 23 (2017) 985-1001.

[7] S. Arora, S. Singh, Butterfly optimization algorithm: a novel approach for global optimization, Soft Computing, 23 (2019) 715-734.

[8] R. Venkata Rao, A. Saroj, A self-adaptive multipopulation-based Jaya algorithm for engineering optimization, Swarm and Evolutionary Computation, 37 (2017) 1-26.

[9] N. Smairi, P. Siarry, K. Ghedira, A hybrid particle swarm approach based on Tribes and tabu search for multi-objective optimization, Optimization Methods and Software, 31 (2016) 204-231.

[10] I. Pence, M.S. Cesmeli, F.A. Senel, B. Cetisli, A new unconstrained global optimization method based on clustering and parabolic approximation, Expert Systems with Applications, 55 (2016) 493507.

[11] R.S. Pavithr, Gursaran, Quantum Inspired Social Evolution (QSE) algorithm for 0-1 knapsack problem, Swarm and Evolutionary Computation, 29 (2016) 33-46.

[12] E. Nabil, A Modified Flower Pollination Algorithm for Global Optimization, Expert Systems with Applications, 57 (2016) 192-203.

[13] S. Mirjalili, A. Lewis, The Whale Optimization Algorithm, Advances in Engineering Software, 95 (2016) 51-67.

[14] Y.-C. Liang, J.R. Cuevas Juarez, A novel metaheuristic for continuous optimization problems: Virus optimization algorithm, Engineering Optimization, 48 (2016) 73-93.

[15] A. Kaveh, V.R. Mahdavi, A new method for modification of ground motions using wavelet transform and enhanced colliding bodies optimization, Applied Soft Computing, 47 (2016) 357-369.

[16] H. Abedinpourshotorban, S. Hasan, S.M. Shamsuddin, N.F. As'Sahra, A differential-based harmony search algorithm for the optimization of continuous problems, Expert Systems with Applications, 62 (2016) 317-332.

[17] Y.-J. Zheng, Water wave optimization: A new nature-inspired metaheuristic, Computers \& Operations Research, 55 (2015) 1-11.

[18] K. Tang, Z. Li, L. Luo, B. Liu, Multi-strategy adaptive particle swarm optimization for numerical 
optimization, Engineering Applications of Artificial Intelligence, 37 (2015) 9-19.

[19] A. Sadollah, H. Eskandar, A. Bahreininejad, J.H. Kim, Water cycle, mine blast and improved mine blast algorithms for discrete sizing optimization of truss structures, Computers \& Structures, 149 (2015) 1-16.

[20] V.K. Patel, V.J. Savsani, Heat transfer search (HTS): a novel optimization algorithm, Information Sciences, 324 (2015) 217-246.

[21] F. Merrikh-Bayat, The runner-root algorithm: A metaheuristic for solving unimodal and multimodal optimization problems inspired by runners and roots of plants in nature, Applied Soft Computing, 33 (2015) 292-303.

[22] B. Javidy, A. Hatamlou, S. Mirjalili, Ions motion algorithm for solving optimization problems, Applied Soft Computing, 32 (2015) 72-79.

[23] M.S. Gonçalves, R.H. Lopez, L.F.F. Miguel, Search group algorithm: A new metaheuristic method for the optimization of truss structures, Computers \& Structures, 153 (2015) 165-184.

[24] A. Mortazavi, V. Togan, Structural Optimization Applying Sine-Cosine Optimization Algorithm, in: International Civil Engineering and Architecture Conference (ICEARC'19), Trabzon, Turkey, 2019.

[25] M. Moloodpoor, A. Mortazavi, N. Ozbalta, Thermal analysis of parabolic trough collectors via a swarm intelligence optimizer, Solar Energy, 181 (2019) 264-275.
[26] M. Kamal, M. Inel, Optimum Design of Reinforced Concrete Continuous Foundation Using Differential Evolution Algorithm, Arabian Journal for Science and Engineering, (2019).

[27] A. Mortazavi, V. Toğan, A. Daloğlu, A. Nuhoğlu, Comparison of Two Metaheuristic Algorithms on Sizing and Topology Optimization of Trusses and Mathematical Functions, Gazi University Journal of Science, 31 (2018) 416-435.

[28] A. Kaveh, A. Dadras, T. Bakhshpoori, Improved thermal exchange optimization algorithm for optimal design of skeletal structures, Smart Structures and Systems, 21 (2018) 263-278.

[29] A. Mortazavi, V. Toğan, A. Nuhoğlu, Interactive search algorithm: A new hybrid metaheuristic optimization algorithm, Engineering Applications of Artificial Intelligence, 71 (2018) 275-292.

[30] Mortazavi, V. Toğan, M. Moloodpoor, Solution of structural and mathematical optimization problems using a new hybrid swarm intelligence optimization algorithm, Advances in Engineering Software, 127 (2019) 106-123.

[31] R.T. Haftka, Requirements for papers focusing on new or improved global optimization algorithms, Structural and Multidisciplinary Optimization, 54 (2016) 1-1.

[32] A. Mortazavi, Interactive fuzzy search algorithm: A new self-adaptive hybrid optimization algorithm, Engineering Applications of Artificial Intelligence, 81 (2019) 270-282. 